\title{
Benign Sublingual Gland Neoplasm
}

National Cancer Institute

\section{Source}

National Cancer Institute. Benign Sublingual Gland Neoplasm. NCI Thesaurus. Code C4601.

A non-metastasizing neoplasm that arises from the sublingual gland. 